\title{
Tanshinone IIA induces TRAIL sensitization of human lung cancer cells through selective ER stress induction
}

\author{
EUN-OK KIM ${ }^{1,2^{*}}$, SHI EUN KANG ${ }^{2 *}$, CHANG RAK IM $^{3^{*}}$, JUN-HEE LEE $^{1,4}$, KWANG SEOK AHN $^{2}$, \\ WOONG MO YANG ${ }^{2}$, JAE-YOUNG UM ${ }^{2}$, SEOK-GEUN LEE ${ }^{1,2}$ and MIYONG YUN ${ }^{2}$ \\ ${ }^{1}$ Korean Medicine Clinical Trial Center, Kyung Hee University Korean Medicine Hospital, \\ ${ }^{2}$ Department of Science in Korean Medicine, ${ }^{3}$ Department of Applied Korean Medicine, \\ ${ }^{4}$ Department of Sasang Constitutional Medicine, Kyung Hee University, Seoul 02447, Republic of Korea
}

Received December 30, 2015; Accepted February 18, 2016

DOI: 10.3892/ijo.2016.3441

\begin{abstract}
Although tumor necrosis factor-related apoptosisinducing ligand (TRAIL) is a promised anticancer medicine targeting only the tumor, most cancers show resistance to TRAIL-induced apoptosis. For this reason, new therapeutic strategies to overcome the TRAIL resistance are required for more effective tumor treatment. In the present study, potential of Tanshinone IIA as a TRAIL sensitizer was evaluated in human non-small cell lung cancer (NSCLC) cells. NSCLC cells showed resistance to TRAIL-mediated cell death, but combination treatment of Tanshinone IIA and TRAIL synergistically decreased cell viability and increased apoptosis in TRAIL-resistant NSCLC cells. Tanshinone IIA greatly induced death receptor 5 (DR5), but not death receptor 4 (DR4). Furthermore, DR5 knockdown attenuated the combination treatment of Tanshinone IIA with TRAILmediated cell death in human NSCLC cells. Tanshinone IIA
\end{abstract}

Correspondence to: Professor Miyong Yun, Department of Science in Korean Medicine, College of Korean Medicine, Kyung Hee University, 26 Kyungheedae-ro, Dongdaemun-gu, Seoul 02447, Republic of Korea

E-mail: myyun91@gmail.com

Professor Seok-Geun Lee, Cancer Preventive Material Development Research Center, Department of Science in Korean Medicine, College of Korean Medicine, Kyung Hee University, 26 Kyungheedae-ro, Dongdaemun-gu, Seoul 02447, Republic of Korea

E-mail: seokgeun@khu.ac.kr

${ }^{*}$ Contributed equally

Abbreviations: TRAIL, tumor necrosis factor-related apoptosisinducing ligand; NSCLC, non-small cell lung cancer; TR, TRAIL-resistant; DR5, death receptor 5; DR4, death receptor 4; CHOP, CCAAT/enhancer-binding protein homologues protein; ER, endoplasmic reticulum; PERK, pancreatic ER kinase; ATF4, activating transcription factor 4; ROS, reactive oxygen species; UPR, unfolded protein response; IRE1, inositol-requiring kinase 1; $\mathrm{BiP}$, polypeptide binding protein; eIF2, eukaryotic initiation factor 2

Key words: Tanshinone IIA, tumor necrosis factor-related apoptosis-inducing ligand, ATF4, CHOP, death receptor 5, endoplasmic reticulum stress, non-small cell lung cancer also increased CHOP and activated the PERK-ATF4 pathway suggesting that Tanshinone IIA increased DR5 and CHOP by activating the PERK-ATF4 pathway. Tanshinone IIA also downregulated phosphorylation of STAT3 and expression of survivin. Taken together, these results indicate that Tanshinone IIA increases TRAIL-induced cell death via upregulating DR5 and downregulating survivin mediated by, respectively, selective activation of PERK/ATF4 and inhibition of STAT3, suggesting combinatorial intervention of Tanshinone IIA and TRAIL as a new therapeutic strategy for human NSCLC.

\section{Introduction}

Lung cancer is the most common cancer with high incidence and mortality worldwide. Approximately $80 \%$ of all lung cancers are non-small cell lung carcinomas (NSCLC) and adenocarcinoma is the most common type (1). Despite increased survival rate via improved diagnosis and treatment of NSCLC, there are still many issues to be resolved including resistance, rapid disease recurrence and progression (2-4). Therefore, new therapeutic strategies to overcome resistance and improve effective treatment of NSCLC patients are urgently required.

The endoplasmic reticulum (ER) participates in various cellular processes, such as protein folding, lipid biosynthesis and calcium storage. Among them precise control of protein folding is a fundamental function of ER to maintain cellular homeostasis $(5,6)$. When misfolded proteins or unfolded proteins are generated during metabolic processes or from various stresses, polypeptide binding protein (BiP or GRP78, sensor) is dissociated from three major ER membrane resident proteins (mediator), inositol-requiring kinase 1 (IRE1), double-strand RNA-activated protein kinase-like ER kinase (PERK) and activating transcription factor 6 (ATF6) leading to activation of the unfolded protein response (UPR) signal (6-9). Dimerization of PERK by UPR activation increases the phosphorylation on Ser 51 of eIF2 leading to attenuation of the global translation initiation (10). On the contrary, eIF2 phosphorylation selectively increases the translation of ATF4 mRNA encoding transcriptional activator of genes involved in the UPR. One of the ATF4 target genes is CCAAT/ enhancer-binding protein homologues protein $(\mathrm{CHOP})$, which is another preferential translation target of eIF2 phosphoryla- 
tion. Increased CHOP during ER stress and UPR activation induces expression of apoptosis-related genes including death receptors DR4 and/or DR5 (11,12). For these reasons, various types of anticancer drugs to modulate proteins involved in the ER stress response have been developed (13).

To develop novel cancer therapeutics to overcome drug resistance, multi-targeting strategies has been recently explored (14). Especially, combination therapy targeting multiple anti-apoptotic proteins and/or pro-apoptotic proteins has been considered effective strategy to overcome TRAIL resistance in most types of cancer including NSCLC and increase the anticancer capacity in treatment of cancers (15). Various compounds isolated from herbal plants have been studied for combination therapy with TRAIL or conventional anticancer drugs (16-20). Particularly, Tanshinone IIA isolated from the dried root of Salvia miltiorrhiza Bunge (Lamiaceae) is one of well-documented natural compounds in terms of combination effects as well as its own anticancer mechanism (21-25). Tanshinone IIA has shown cytotoxic effects in several types of human cancer cells in vitro and in vivo $(26,27)$.

A recent study revealed that Tanshinone IIA induces apoptosis through inhibition of JAK/STAT3 signaling in myeloid leukemia cells (28). In the present study, we focused on antitumor effects of Tanshinone IIA to sensitize TRAIL-mediated apoptosis in human NSCLC cells through modulation of ER stress response and apoptosis signals.

\section{Materials and methods}

Cell culture and reagents. NSCLC cell lines were obtained from the American Type Culture Collection (ATCC; Manassas, VA, USA). A549, H596, H1299, Calu-1 and H460 cell lines were grown in RPMI-1640 medium (HyClone Laboratories, Logan, UT, USA) containing $10 \%$ heat-inactivated fetal bovine serum (FBS) and $1 \%$ antibiotics (HyClone Laboratories) in a humidified atmosphere of $95 \%$ air and $5 \% \mathrm{CO}_{2}$ at $37^{\circ} \mathrm{C}$, and the viability of cultured cells was monitored by a LUNA-FL automated cell counter (Logos Biosystems, Anyang, Gyeonggi-do, Republic of Korea). Tanshinone IIA (M.W.=328, T4952, $\geq 97 \%$-HPLC) was purchased from Sigma Aldrich (St. Louis, MO, USA).

MTT assay. Cytotoxicity of Tanshinone IIA, TRAIL or combination with Tanshinone IIA and TRAIL was evaluated by 3-(4,5-dimethylthiazol-2-yl)-2,5-diphenyltetrazolium bromide (MTT; Sigma Chemical) assay in NSCLCs. Cells were seeded in a 96-well-microplate at a density of $1 \times 10^{4}$ cells/ well and treated with various concentrations of Tanshinone IIA. After the indicated time, the medium was removed, and fresh medium containing $1 \mathrm{mg} / \mathrm{ml}$ MTT was added to each well. The cells were incubated at $37^{\circ} \mathrm{C}$ for $2 \mathrm{~h}$ and then an equal volume of MTT lysis buffer was added to each well. The cells were incubated at $37^{\circ} \mathrm{C}$ overnight. Optical density (OD) was measured using a microplate reader (Tecan Austria $\mathrm{GmbH}$, Grödig, Austria) at $570 \mathrm{~nm}$.

Western blotting. Whole cell lysates were prepared, and western blotting was performed as previously described (28). In brief, total proteins from A549, H596, H1299 and Calu-1 cells were extracted with RIPA buffer $(50 \mathrm{mM}$ Tris- $\mathrm{HCl}$, pH 7.4, $150 \mathrm{mM} \mathrm{NaCl}, 1 \%$ NP-40, $0.25 \%$ deoxycholic acid-
$\mathrm{Na}, 1 \mathrm{mM}$ EDTA, $1 \mathrm{mM} \mathrm{Na} \mathrm{VO}_{4}, 1 \mathrm{mM} \mathrm{NaF}$ ) including protease inhibitor cocktail (Roche). Primary antibodies for CHOP, DR5, ATF4, GRP78, calnexin, PERK, PDI, IRE-1 $\alpha$ (Cell Signaling Technology, Beverly, MA, USA), DR4, PARP, cleaved caspase-3, caspase-3, survivin (Santa Cruz Biotechnology, Santa Cruz, CA, USA), anti- $\beta$-actin (SigmaAldrich) and secondary antibodies coupled to horseradish peroxidase (HRP) from Vector Laboratories Inc. (Burlingame, CA, USA) were used for immunoblotting. Expression was visualized by using enhanced chemiluminescence (ECL) Western blotting detection reagent (Amersham Pharmacia, Piscataway, NJ, USA).

Reverse transcription-polymerase chain reaction (RT-PCR). Total RNA was isolated from the NSCLC cells with the TRIzol reagent (Invitrogen, Carlsbad, CA, USA) according to the manufacturer's instructions. Total RNA $(2 \mu \mathrm{g})$ isolated from cells was reverse transcribed to cDNA using oligo-dT and random primers. Reverse transcription was done using a thermal program of $50^{\circ} \mathrm{C}$ for $60 \mathrm{~min}$ and $85^{\circ} \mathrm{C}$ for $5 \mathrm{~min}$. The cDNA was amplified by PCR using the following specific primers: DR4 (forward, 5'-GGCTGAGGACAATGCTCACA-3' and reverse, 5'-TTGCTGCTCAGAGACGAAAGTG'); DR5 (forward, 5'-GACTCTGAGACAGTGCTTCGATGA-3' and reverse, 5'-CCATGAGGCCCAACTTCCT-3'); CHOP (forward, 5'-CAACTGCAGAGAATTCAGCTGA-3' and reverse, 5'-AC TGATGCTCTAGATTGTTCAT-3'); GAPDH (forward, 5'-CC ACTCCTCCACCTTTGAC-3' and reverse, 5'-ACCCTGTTG CTGTAGCCA-3'); XBP-1 (forward, 5'-CTGGAACAGCAA GTGGTAGA-3' and reverse, 5'-CTGGGTCCTTCTGGGTA GAC-3'). All primers were synthesized by Bioneer, Co. (Daejeon, Republic of Korea).

Apoptosis assay. NSCLCs were seeded in 6-well plates, treated with indicated compounds for $24 \mathrm{~h}$ and stained with Annexin V-fluorescein isothiocyanate (FITC) and propidium iodide (PI) kit (BioVision Technologies Inc., Golden, CO, USA). The cells were then analyzed using fluorescenceactivated cell sorting FACSCalibur flow cytometry.

siRNA transfection. Transfection of control or DR5 siRNA (Bioneer) was performed using transfection reagent INTERFERin (Polyplus-transfection, New York, NY, USA) according to the manufacturer's protocol.

Validation of synergy between tanshinone IIA and TRAIL. To determine the synergy between Tanshinone IIA and TRAIL, cytotoxicity assay was performed in four NSCLS cell lines by the gradual increase with a same constant ratio between Tanshinone IIA and TRAIL. The values from viability assays were analyzed by CalcuSyn software (Biosoft, Ferguson, MO, USA). The fraction of living cells in each concentration was applied for the analysis of synergism between Tanshinone IIA and TRAIL by CalcuSyn software.

Statistical analysis. Data are presented as mean \pm standard deviation (SD) from at least three independent experiments in triplicate and analyzed for statistical significance using the unpaired Student's t-test. $\mathrm{P}<0.05$ was considered statistically significant. 

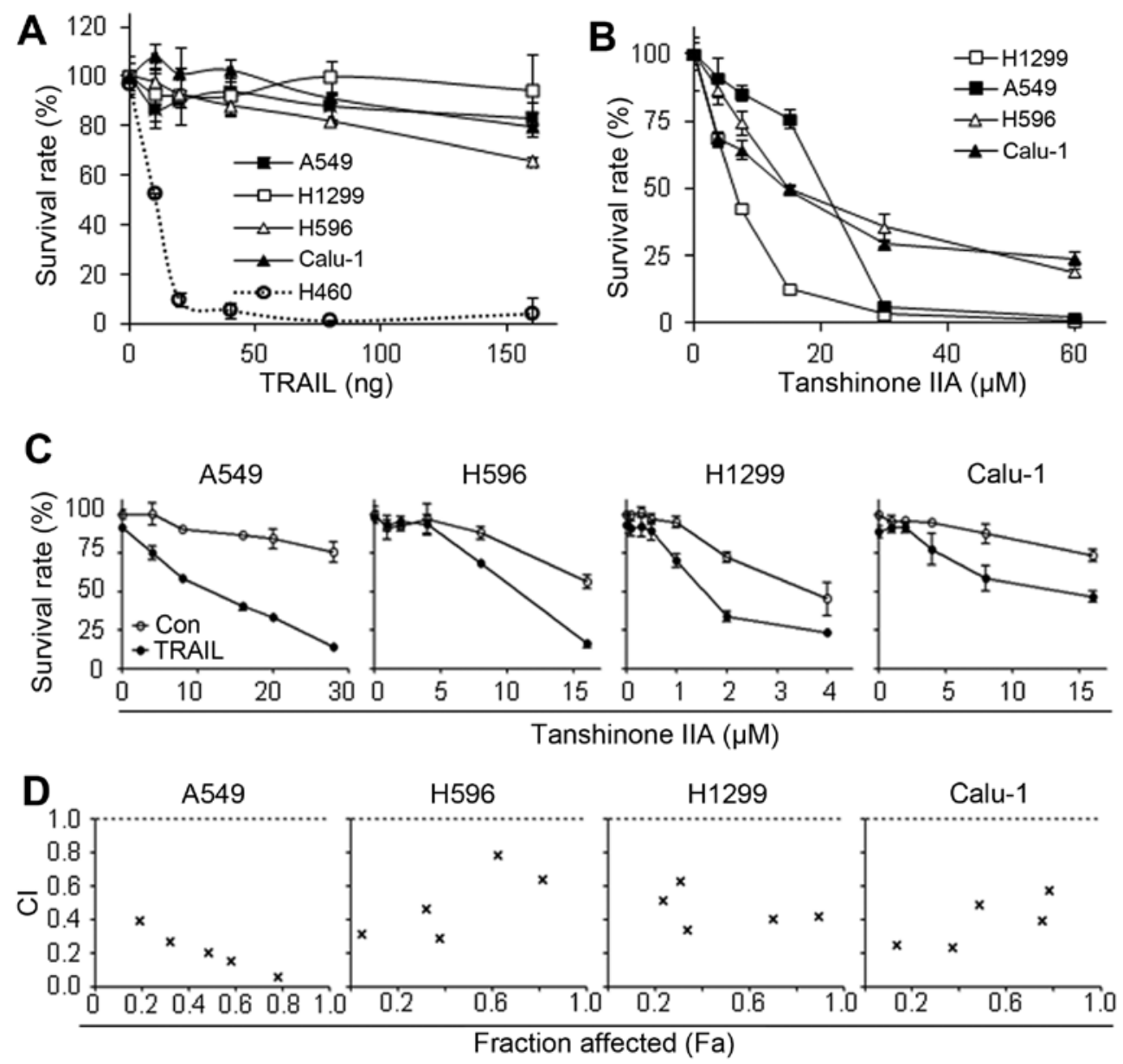

Figure 1. Synergistic effect of tanshinone IIA and TRAIL on cytotoxicity of human TRAIL-resistant NSCLC cells. (A and B) NSCLC cells were treated with TRAIL and/or tanshinone IIA for $24 \mathrm{~h}$ as indicated. (C) NSCLC cells were treated with $25 \mathrm{ng} / \mathrm{ml}$ of TRAIL and/or tanshinone IIA for $24 \mathrm{~h}$ as indicated. Cell viability was then analyzed by the MTT assays. (D) Combination index (CI) values with fraction affected (Fa) between Tanshinone IIA and TRAIL in human NSCLC cells were calculated using Calcusyn software.

\section{Results}

Combination treatment of Tanshinone IIA and TRAIL synergistically increases the cytotoxicity in TRAIL-resistant NSCLCs. In order to investigate the possible therapeutic effect of Tanshinone IIA and TRAIL in NSCLC, we first examined whether single treatment of Tanshinone IIA or TRAIL affects viability of human NSCLC cells. Consistent with previous reports (29,30), A549, H596, H1299 and Calu-1 showed high resistance to TRAIL, while H460 was very sensitive to TRAIL with $\sim 12 \mathrm{ng} / \mathrm{ml} \mathrm{IC}_{50}$ (Fig. 1A). Tanshinone IIA was cytotoxic in all the tested NSCLC cell lines with $\mathrm{IC}_{50}$ values of $\sim 10-30 \mu \mathrm{M}$ (Fig. 1B). Furthermore, in evaluation of combination effect of Tanshinone IIA and TRAIL, co-treatment synergistically decreased cell viability (Fig. 1C). Surprisingly, the combination index $(\mathrm{CI})$ values below 0.8 at all fractions affected $(\mathrm{Fa})$ points, suggesting that the combination treatment showed strong synergy in all four tested TRAIL resistant NSCLC cell lines (Fig. 1D).

Combination treatment effectively induces apoptosis in TRAIL-resistant NSCLCs. Canonical cell death signals through TRAIL receptors trigger extrinsic apoptosis which occurred by extracellular death signals (31). To verify whether synergistic effect of Tanshinone IIA on cytotoxicity is mediated by apoptotic cell death, we analyzed apoptosis in Tanshinone IIA and TRAIL co-treated NSCLC cells. As shown in Fig. 2A, Tanshinone IIA/TRAIL combination treatment intensively upregulated cleaved-caspase-3, -8 and cleaved-PARP levels, and even combination with half dose of each single treatment showed more increased cleaved forms indicating induction of apoptosis. Consistently, Annexin V and PI double staining revealed that apoptotic population was markedly increased in co-treated NSCLC cells compared to control or single treated A549 and H1299 cells (Fig. 2B). These data indicate that Tanshinone IIA may be a novel TRAIL sensitizer in NSCLC cells.

Tanshinone IIA-mediated cell death is through DR5 induction, but not DR4 in TRAIL-resistant NSCLCs. Cancer cells usually acquire TRAIL resistance via downregulation of DR4 and/or DR5 $(32,33)$. To confirm if Tanshinone IIA overcomes the TRAIL resistance by modulation of death receptors, we evaluated the expression of the proteins. Tanshinone IIA treatment significantly increased DR5, but not DR4 in A549, H596 and H1299 cells (Fig. 3A). Since CHOP is one of major transcriptional activators of DR5 $(34,35)$, we next examined the effect of Tanshinone IIA on the expression of CHOP. As shown 

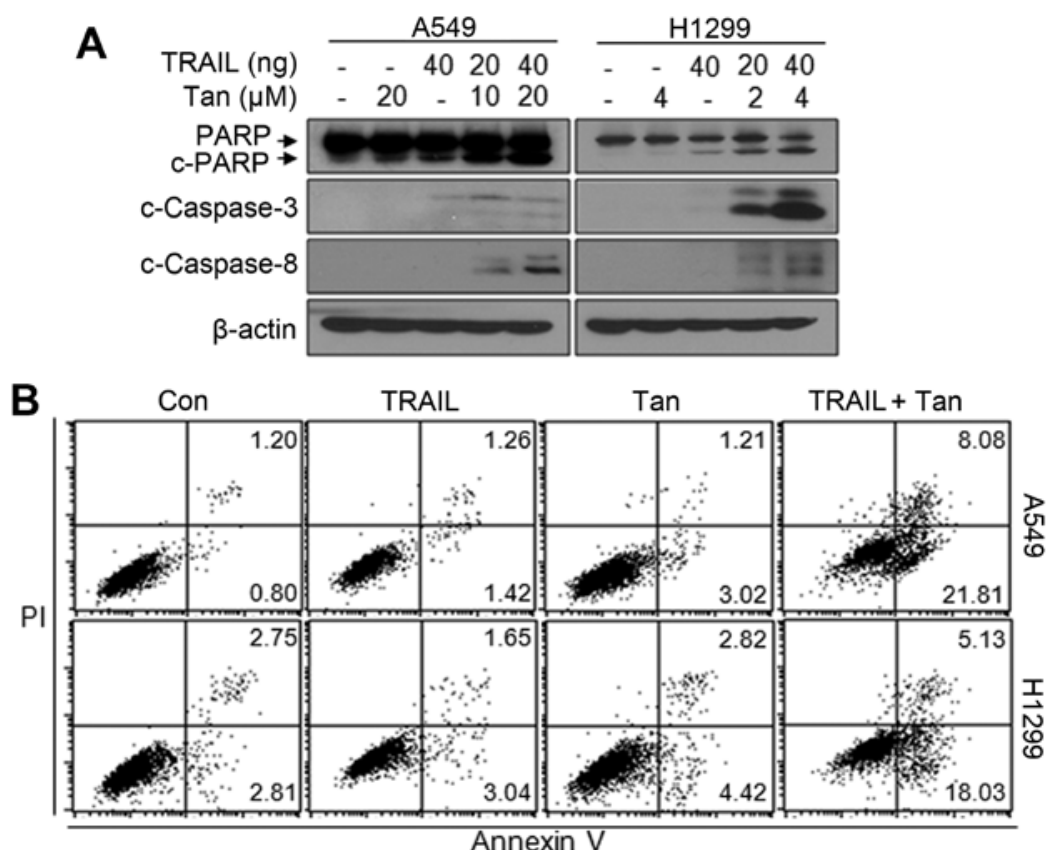

Figure 2. Synergistic effect of Tanshinone IIA and TRAIL on apoptosis induction in human TRAIL-resistant NSCLC cells. (A) Cells were treated with Tanshinone IIA and TRAIL for $24 \mathrm{~h}$ as indicated. Cells lysates were prepared and subjected to western blotting with the indicated antibodies. $\beta$-actin was used as an internal standard. (B) Cells were treated with Tanshinone IIA and/or TRAIL as following: A549 cells with $10 \mu \mathrm{M}$ of Tanshinone IIA/40 ng/ml of TRAIL and $\mathrm{H} 1299$ cells with $4 \mu \mathrm{M}$ of Tanshinone IIA/ $40 \mathrm{ng} / \mathrm{ml}$ of TRAIL. After staining with Annexin V-FITC and PI, the apoptotic cells were analyzed by a dot plot using a flow cytometer. The numbers in each plot indicate the percentage of apoptotic cells.

A
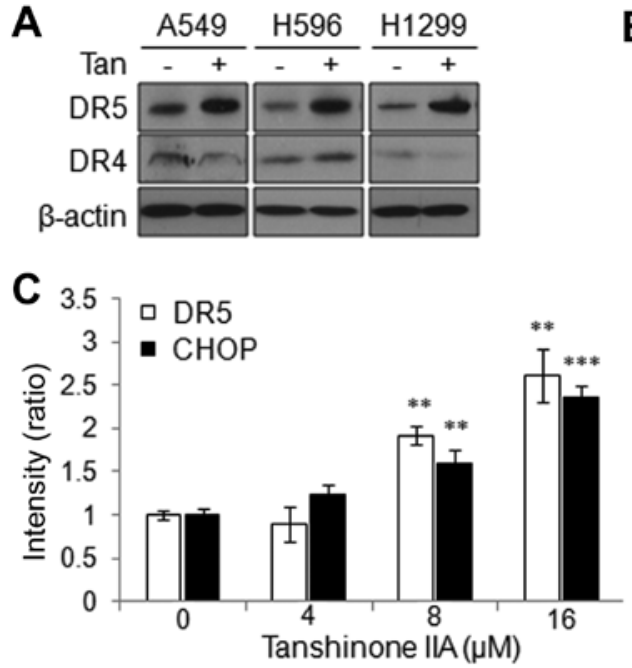

$\mathbf{E}$

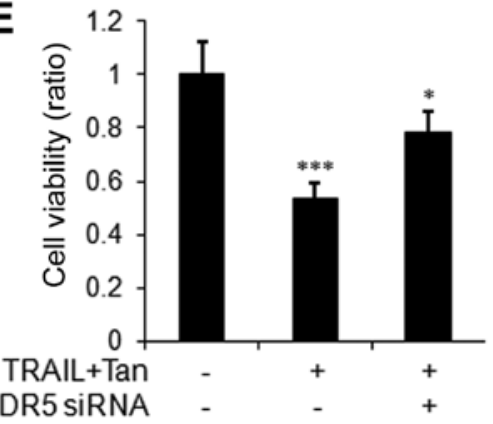

B $\operatorname{Tan}(\mu \mathrm{M}) \quad 0 \quad 4 \quad 8 \quad 16$

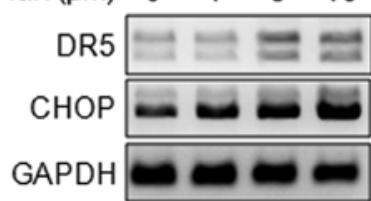

D TRAIL+Tan $-+\quad++$
DR5 SiRNA $-\frac{+}{-}+$

DR5

$\beta$-actin

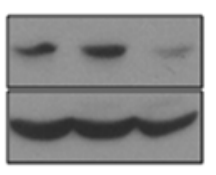

Figure 3. Effect of Tanshinone IIA on the expression of DR5 in human TRAIL-resistant NSCLC cells. (A) A549, H596 and H1299 cells were treated with 20 , 20 and $4 \mu \mathrm{M}$ of Tanshinone IIA, respectively. Total cell protein extracts were subjected to immunoblotting with the indicated antibodies. $\beta$-actin was used as an internal control. (B) A549 cells were treated with Tanshinone IIA for $24 \mathrm{~h}$ as indicated. Total RNA was isolated and RT-PCR analysis was performed as described in Materials and methods. (C) Representation of quantified band intensity in B. (D and E) A549 cells were transfected with DR5 siRNA or control siRNA. Forty-eight hours after transfection, cells were treated with $20 \mu \mathrm{M}$ of Tanshinone IIA and $10 \mathrm{ng} / \mathrm{ml}$ of TRAIL for $24 \mathrm{~h}$, and (D) the cell lysates were subjected to immunoblotting with the indicated antibodies and (E) the viability was then analyzed by the MTT assays. ${ }^{* * *} \mathrm{P}<0.001$ and ${ }^{*} \mathrm{P}<0.05$ compared to control and TR/Tan, respectively. (TR, TRAIL; Tan, Tanshinone IIA). 


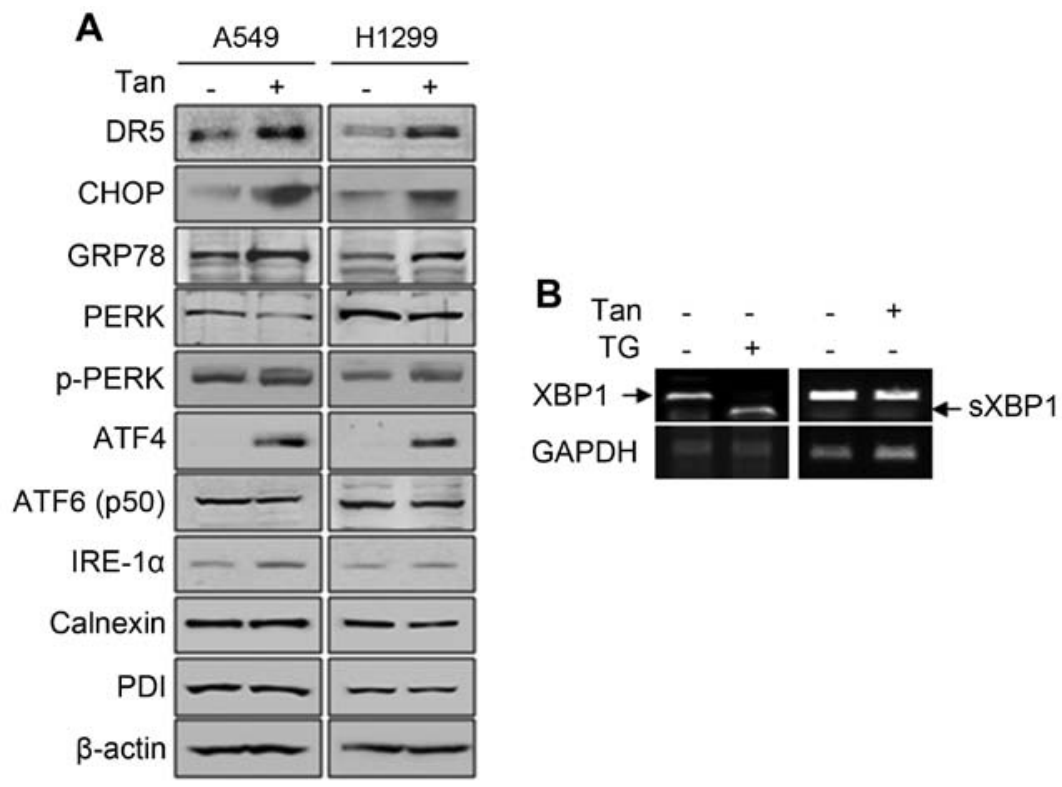

Figure 4. Effect of Tanshinone IIA on the ER stress signaling pathways in human TRAIL-resistant NSCLC cells. (A) A549 and H1299 cells were treated with $20 \mu \mathrm{M}$ of Tanshinone IIA for $24 \mathrm{~h}$. Cells lysates were subjected to western blotting with the indicated antibodies. (B) A549 cells were treated with $20 \mu \mathrm{M}$ of Tanshinone IIA (Tan) for $24 \mathrm{~h}$ or thapsigargin (Tg) for $8 \mathrm{~h}$. Total RNA was isolated and RT-PCR was performed to detect the spliced form (sXBP-1) and unspliced form of XBP-1 mRNA. PCR products were separated on a $3 \%$ agarose gel.

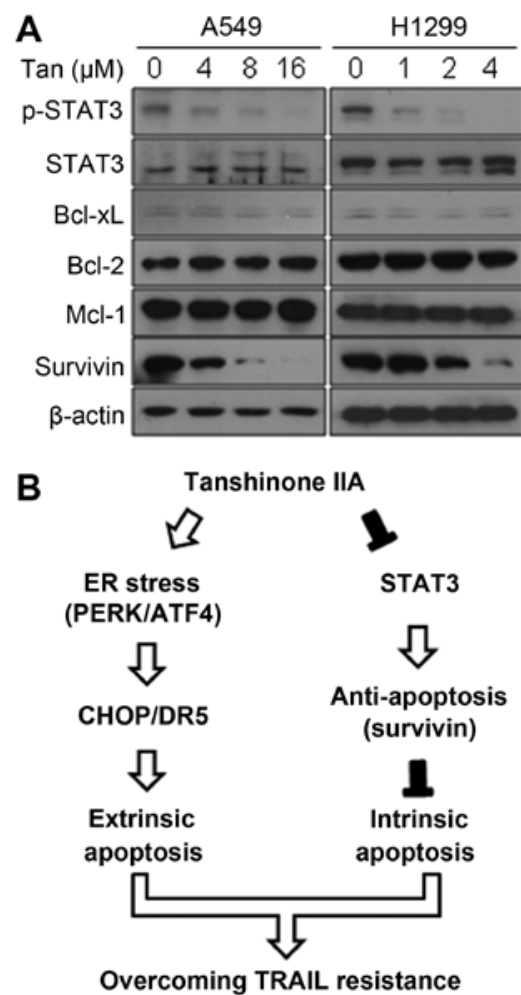

Figure 5. Effect of Tanshinone IIA on STAT3 and anti-apototic molecules. (A) A549 and H1299 cells were treated with Tanshinone IIA for $24 \mathrm{~h}$ as indicated. Whole cell extracts were prepared and analysed by western blotting using indicated antibodies. $\beta$-actin was used as the internal standard. (B) Schematic diagram indicating the effect of Tanshinone IIA on ER stress and STAT3 in NSCLC cells.

in Fig. 3B and C, Tanshinone IIA transcriptionally induced CHOP and also DR5 in a dose-dependent manner. In order to confirm the role of DR5 in the Tanshinone IIA-mediated
TRAIL sensitization of NSCLC cells, we depleted DR5 with specific siRNA (Fig. 3D). As shown in Fig. 3E, inhibition of DR5 restored the TRAIL and Tanshinone IIA-mediated decrease of cell viability. These results indicated that Tanshinone IIA significantly increased expression of DR5, leading to increase of TRAIL sensitivity in human NSCLC cells.

Tanshinone IIA selectively induces the PERK/ATF4 pathway of ER stress response signals causing induction of DR5. Since $\mathrm{CHOP}$ is transcriptionally induced by ER stress response signals (36), we then examined whether Tanshinone IIA can regulate signaling molecules involved in ER stress response in NSCLCs. As shown in Fig. 4A, Tanshinone IIA significantly induced GRP78 which is an ER stress sensor protein. Tanshinone IIA also activated the PERK/ATF4 signal which is one of three ER stress inducers, but the other two pathways, ATF6 and IRE-1 $\alpha$ signals, were not affected at all (Fig. 4A). In addition, Tanshinone IIA did not induce the splicing of XBP1, while treatment of a well-known ER stress inducer thapsigargin (TG) greatly increased the spliced form of XBP1 mRNA (Fig. 4B). Furthermore, Tanshinone IIA did not affect the expression of other ER resident chaperons contributing protein folding (Fig. 4A). These data indicate that Tanshinone IIA increases DR5 and CHOP by selective activation of the PERK/ATF4 signal.

Tanshinone IIA suppresses STAT3 and its downstream target survivin. Because tumor cells with TRAIL resistance usually possess increased levels of anti-apoptotic proteins $(37,38)$, these proteins have been regarded major targets for development of cancer therapeutics. As shown in Fig. 5, Tanshinone IIA decreased phosphorylation of STAT3, which is known to induce anti-apoptotic proteins including survivin, while total STAT3 expression was not changed at all. Furthermore, Tanshinone IIA suppressed survivin in a dose-dependent 
manner (Fig. 5). However, other anti-apoptotic proteins Bcl-xL, Bcl-2 and Mcl-1 were not affected by Tanshinone IIA (Fig. 5A). These results suggest that Tanshinone IIA inhibits the STAT3 pathway leading to suppression of survivin in human NSCLC cells.

\section{Discussion}

Even though activation of the TRAIL receptor pathway is a promising therapeutic strategy to selectively remove cancer cells, most cancers including NSCLC have various ways to evade the TRAIL-mediated cell death. Therefore, combination treatment strategies to overcome TRAIL resistance have been intensively studied. Particularly, herbal compounds targeting death receptors are considered effective interventions to increase the TRAIL sensitivity in tumor cells. Recent studies have proven that several natural compounds such as cycloanthranilylproline, cryptotanshinone, kurarinone, curcumin and rocaglamide increased the TRAIL sensitivity in various types of cancer resistant to TRAIL (39-43).

In the present study, we evaluated whether another herbal compound, tanshinone IIA can overcome the TRAIL resistance in human NSCLC cells. Consistent with previous reports $(44,45)$, tanshinone IIA significantly increased DR5 through CHOP induction (Fig. 3). Furthermore, we newly revealed that Tanshinone IIA selectively activated the PERK/ATF4 pathway (Fig. 4). These results suggest that Tanshinone IIA induces DR5 by increasing CHOP through selective activation of the PERK/ATF4 pathway, one of the ER stress response signals. Reactive oxygen species (ROS), chemically reactive molecules, play roles in regulating cell signaling and homeostasis (46). ROS is generated during oxygen usage in all cellular compartments, such as the mitochondrial respiratory chain and ER (47) suggesting ROS as one of ER stress inducers. Based on our previous report, decursin has also similar functions, such as induction of selective ER stress via ROS generation and TRAIL sensitivity in lung cancer cells (18). Recent report indicated that Tanshinone IIA induces considerable amount of ROS formation in A549, but not H596 cells, which lack the NAD(P)H:quinone oxidoreductase (NQO1) (48). Consistently, we also detected ER stress response signals in Tanshinone IIA-treated A549 cells, but not in H596 (data not shown), indicating that ER stress induction by Tanshinone IIA may be mediated by ROS generation. However, more detailed molecular mechanisms how Tanshinone IIA induces synergistic cytotoxic effect with TRAIL in H596 cells should be further elucidated.

Because most tumors are resistant to TRAIL, targeting the main factors associated with the TRAIL resistance is a common strategy to effectively increase the TRAIL sensitivity in treating cancer. For example, inhibition of cell survival factors including survivin and/or induction of anti-apoptotic factors have been used for overcoming the TRAIL resistance (49-55). STAT3 is a well-known transcription factor regulating genes encoding proteins involved in cell survival including survivin and Bcl-2. Major function of STAT3 is to convey signals from the cell surface to the nucleus on activation by cytokines and growth factors $(56,57)$. Phosphorylation of STAT3 by activation of receptor tyrosine kinases (RTKs), such as EGFR, leads to its dimerization and then translocation to the nucleus. Numerous studies have proved that hyperphosphorylated STAT3 are widely detected in various types of human tumor specimens and is required for malignant transformation of cultured cells (58-62). In this regard, diverse novel STAT3 inhibitors have been developed $(60,63)$. Tanshinone IIA significantly downregulated phosphorylation of STAT3, probably leading to suppression of survivin in human NSCLC cells (Fig. 5).

Taken together, these results clearly demonstrate that Tanshinone IIA selectively induces the ER stress, PERK/ATF4/CHOP pathway leading to significant induction of DR5 and reduces survivin via inactivating STAT3. Furthermore, combination treatment of Tanshinone IIA and TRAIL showed synergistic induction of apoptotic cell death in human NSCLC cells resistant to TRAIL (Fig. 5B), suggesting that Tanshinone IIA is a promising TRAIL sensitizer in NSCLCs and that combination of Tanshinone IIA with TRAIL would be a good therapeutic strategy for treating NSCLCs.

\section{Acknowledgements}

The present study was supported by the research grants (NRF-2014R1A1A2057918, NRF-2014R1A1A2056230 and NRF-2015R1A4A1042399) from the National Research Foundation of Korea.

\section{References}

1. Mitsudomi T: Advances in target therapy for lung cancer. Jpn $\mathbf{J}$ Clin Oncol 40: 101-106, 2010.

2. Sun JM, Choi YL, Ji JH, Ahn JS, Kim KM, Han J, Ahn MJ and Park K: Small-cell lung cancer detection in never-smokers: Clinical characteristics and multigene mutation profiling using targeted next-generation sequencing. Ann Oncol 26: 161-166, 2015.

3. Xu C, Zhou Q and Wu YL: Can EGFR-TKIs be used in first line treatment for advanced non-small cell lung cancer based on selection according to clinical factors? - A literature-based metaanalysis. J Hematol Oncol 5: 62, 2012.

4. Zheng DJ, Yu GH, Gao JF and Gu JD: Concomitant EGFR inhibitors combined with radiation for treatment of non-small cell lung carcinoma. Asian Pac J Cancer Prev 14: 4485-4494, 2013.

5. Schubert U, Antón LC, Gibbs J, Norbury CC, Yewdell JW and Bennink JR: Rapid degradation of a large fraction of newly synthesized proteins by proteasomes. Nature 404: 770-774, 2000.

6. Yewdell JW and Nicchitta CV: The DRiP hypothesis decennial: Support, controversy, refinement and extension. Trends Immunol 27: 368-373, 2006.

7. Schröder M and Kaufman RJ: ER stress and the unfolded protein response. Mutat Res 569: 29-63, 2005.

8. van Anken E and Braakman I: Endoplasmic reticulum stress and the making of a professional secretory cell. Crit Rev Biochem Mol Biol 40: 269-283, 2005.

9. van der Vlies D, Makkinje M, Jansens A, Braakman I, Verkleij AJ, Wirtz KW and Post JA: Oxidation of ER resident proteins upon oxidative stress: Effects of altering cellular redox/antioxidant status and implications for protein maturation. Antioxid Redox Signal 5: 381-387, 2003.

10. Shi Y, Vattem KM, Sood R, An J, Liang J, Stramm L and Wek RC: Identification and characterization of pancreatic eukaryotic initiation factor 2 alpha-subunit kinase, PEK, involved in translational control. Mol Cell Biol 18: 7499-7509, 1998.

11. Wek RC and Cavener DR: Translational control and the unfolded protein response. Antioxid Redox Signal 9: 2357-2371, 2007.

12. Xu L, Su L and Liu X: PKC $\delta$ regulates death receptor 5 expression induced by PS-341 through ATF4-ATF3/CHOP axis in human lung cancer cells. Mol Cancer Ther 11: 2174-2182, 2012. 
13. Yadav RK, Chae SW, Kim HR and Chae HJ: Endoplasmic reticulum stress and cancer. J Cancer Prev 19: 75-88, 2014.

14. Yap TA, Omlin A and de Bono JS: Development of therapeutic combinations targeting major cancer signaling pathways. J Clin Oncol 31: 1592-1605, 2013

15. Wang S: The promise of cancer therapeutics targeting the TNF-related apoptosis-inducing ligand and TRAIL receptor pathway. Oncogene 27: 6207-6215, 2008

16. Frew AJ, Lindemann RK, Martin BP, Clarke CJ, Sharkey J, Anthony DA, Banks KM, Haynes NM, Gangatirkar P, Stanley K, et al: Combination therapy of established cancer using a histone deacetylase inhibitor and a TRAIL receptor agonist. Proc Natl Acad Sci USA 105: 11317-11322, 2008.

17. Hellwig CT and Rehm M: TRAIL signaling and synergy mechanisms used in TRAIL-based combination therapies. Mol Cancer Ther 11: 3-13, 2012

18. Kim J, Yun M, Kim EO, Jung DB, Won G, Kim B, Jung JH and Kim SH: Generation of ROS by Decursin selectively induces the ER stress pathway components ATF4/PERK leading to the synergistic enhancement of TRAIL-induced apoptosis. Br J Pharmacol: Dec 11, 2015. doi: 10.1111/bph.13408 (Epub ahead of print).

19. Matsuzaki H, Schmied BM, Ulrich A, Standop J, Schneider MB, Batra SK, Picha KS and Pour PM: Combination of tumor necrosis factor-related apoptosis-inducing ligand (TRAIL) and actinomycin D induces apoptosis even in TRAIL-resistant human pancreatic cancer cells. Clin Cancer Res 7: 407-414, 2001.

20. Park D, Ha IJ, Park SY, Choi M, Lim SL, Kim SH, Lee JH, Ahn KS, Yun M and Lee SG: Morusin induces TRAIL sensitization by regulating EGFR and DR5 in human glioblastoma cells. J Nat Prod: Feb 1, 2016 (Epub ahead of print).

21. Chiu SC, Huang SY, Chang SF, Chen SP, Chen CC, Lin TH, Liu HH, Tsai TH, Lee SS, Pang CY, et al: Potential therapeutic roles of tanshinone IIA in human bladder cancer cells. Int J Mol Sci 15: 15622-15637, 2014.

22. Chiu TL and Su CC: Tanshinone IIA induces apoptosis in human lung cancer A549 cells through the induction of reactive oxygen species and decreasing the mitochondrial membrane potential. Int J Mol Med 25: 231-236, 2010.

23. Jiao JW and Wen F: Tanshinone IIA acts via p38 MAPK to induce apoptosis and the down-regulation of ERCC1 and lung-resistance protein in cisplatin-resistant ovarian cancer cells. Oncol Rep 25: 781-788, 2011.

24. Su CC and Lin YH: Tanshinone IIA inhibits human breast cancer cells through increased Bax to Bcl-xL ratios. Int J Mol Med 22: 357-361, 2008

25. Tseng PY, Lu WC, Hsieh MJ, Chien SY and Chen MK: Tanshinone IIA induces apoptosis in human oral cancer KB cells through a mitochondria-dependent pathway. BioMed Res Int 2014: 540516, 2014

26. Jeon YJ, Kim JS, Hwang GH, Wu Z, Han HJ, Park SH, Chang W, Kim LK, Lee YM, Liu KH, et al: Inhibition of cytochrome P450 $2 \mathrm{~J} 2$ by tanshinone IIA induces apoptotic cell death in hepatocellular carcinoma HepG2 cells. Eur J Pharmacol 764: 480-488, 2015.

27. Munagala R, Aqil F, Jeyabalan J and Gupta RC: Tanshinone IIA inhibits viral oncogene expression leading to apoptosis and inhibition of cervical cancer. Cancer Lett 356: 536-546, 2015.

28. Jung JH, Kwon TR, Jeong SJ, Kim EO, Sohn EJ, Yun M and Kim SH: Apoptosis induced by Tanshinone IIA and cryptotanshinone is mediated by distinct JAK/STAT3/5 and SHP1/2 signaling in chronic myeloid leukemia K562 cells. Evid Based Complement Alternat Med 2013: 805639, 2013

29. Jin CY, Moon DO, Lee JD, Heo MS, Choi YH, Lee CM, Park YM and Kim GY: Sulforaphane sensitizes tumor necrosis factorrelated apoptosis-inducing ligand-mediated apoptosis through downregulation of ERK and Akt in lung adenocarcinoma A549 cells. Carcinogenesis 28: 1058-1066, 2007.

30. La Monica S, Galetti M, Alfieri RR, Cavazzoni A, Ardizzoni A, Tiseo M, Capelletti M, Goldoni M, Tagliaferri S, Mutti A, et al: Everolimus restores gefitinib sensitivity in resistant non-small cell lung cancer cell lines. Biochem Pharmacol 78: 460-468, 2009.

31. Wallach D, Varfolomeev EE, Malinin NL, Goltsev YV, Kovalenko AV and Boldin MP: Tumor necrosis factor receptor and Fas signaling mechanisms. Annu Rev Immunol 17: 331-367, 1999.
32. Ozören N, Fisher MJ, Kim K, Liu CX, Genin A, Shifman Y, Dicker DT, Spinner NB, Lisitsyn NA and El-Deiry WS: Homozygous deletion of the death receptor DR4 gene in a nasopharyngeal cancer cell line is associated with TRAIL resistance. Int J Oncol 16: 917-925, 2000.

33. Wang S and El-Deiry WS: Inducible silencing of KILLER/DR5 in vivo promotes bioluminescent colon tumor xenograft growth and confers resistance to chemotherapeutic agent 5-fluorouracil. Cancer Res 64: 6666-6672, 2004.

34. Lee JY, Jung KH, Morgan MJ, Kang YR, Lee HS, Koo GB, Hong SS, Kwon SW and Kim YS: Sensitization of TRAILinduced cell death by 20 (S)-ginsenoside $\mathrm{Rg} 3$ via CHOP-mediated DR5 upregulation in human hepatocellular carcinoma cells. Mol Cancer Ther 12: 274-285, 2013

35. Oh YT, Liu X, Yue P, Kang S, Chen J, Taunton J, Khuri FR and Sun SY: ERK/ribosomal S6 kinase (RSK) signaling positively regulates death receptor 5 expression through co-activation of CHOP and Elk1. J Biol Chem 285: 41310-41319, 2010.

36. Yamaguchi $\mathrm{H}$ and Wang HG: CHOP is involved in endoplasmic reticulum stress-induced apoptosis by enhancing DR5 expression in human carcinoma cells. J Biol Chem 279: 45495-45502, 2004.

37. Ackler S, Mitten MJ, Chen J, Clarin J, Foster K, Jin S, Phillips DC, Schlessinger S, Wang B, Leverson JD, et al: Navitoclax (ABT-263) and bendamustine \pm rituximab induce enhanced killing of non-Hodgkin's lymphoma tumours in vivo. Br J Pharmacol 167: 881-891, 2012

38. Wang G, Zhan Y, Wang H and Li W: ABT-263 sensitizes TRAILresistant hepatocarcinoma cells by downregulating the Bcl-2 family of anti-apoptotic protein. Cancer Chemother Pharmacol 69: 799-805, 2012

39. Bleumink M, Köhler R, Giaisi M, Proksch P, Krammer PH and Li-Weber M: Rocaglamide breaks TRAIL resistance in HTLV1-associated adult T-cell leukemia/lymphoma by translational suppression of c-FLIP expression. Cell Death Differ 18: 362-370, 2011.

40. Hasegawa H, Yamada Y, Komiyama K, Hayashi M, Ishibashi M, Sunazuka T, Izuhara T, Sugahara K, Tsuruda K, Masuda M, et al: A novel natural compound, a cycloanthranilylproline derivative (Fuligocandin B), sensitizes leukemia cells to apoptosis induced by tumor necrosis factor related apoptosis-inducing ligand (TRAIL) through 15-deoxy-Delta ${ }^{12,14}$ prostaglandin $\mathrm{J}_{2}$ production. Blood 110: 1664-1674, 2007.

41. Park S, Cho DH, Andera L, Suh N and Kim I: Curcumin enhances TRAIL-induced apoptosis of breast cancer cells by regulating apoptosis-related proteins. Mol Cell Biochem 383: 39-48, 2013.

42. Seo OW, Kim JH, Lee KS, Lee KS, Kim JH, Won MH, Ha KS, Kwon YG and Kim YM: Kurarinone promotes TRAIL-induced apoptosis by inhibiting NF- $\kappa \mathrm{B}$-dependent cFLIP expression in HeLa cells. Exp Mol Med 44: 653-664, 2012.

43. Tse AK, Chow KY, Cao HH, Cheng CY, Kwan HY, Yu H, Zhu GY, Wu YC, Fong WF and Yu ZL: The herbal compound cryptotanshinone restores sensitivity in cancer cells that are resistant to the tumor necrosis factor-related apoptosis-inducing ligand. J Biol Chem 288: 29923-29933, 2013.

44. Chang CC, Kuan CP, Lin JY, Lai JS and Ho TF: Tanshinone IIA facilitates TRAIL sensitization by up-regulating DR5 through the ROS-JNK-CHOP signaling Axis in human ovarian carcinoma cell lines. Chem Res Toxicol 28: 1574-1583, 2015.

45. Lin JY, Ke YM, Lai JS and Ho TF: Tanshinone IIA enhances the effects of TRAIL by downregulating survivin in human ovarian carcinoma cells. Phytomedicine 22: 929-938, 2015.

46. Devasagayam TP, Tilak JC, Boloor KK, Sane KS, Ghaskadbi SS and Lele RD: Free radicals and antioxidants in human health: Current status and future prospects. J Assoc Physicians India 52: 794-804, 2004

47. Stadtman ER: Importance of individuality in oxidative stress and aging. Free Radic Biol Med 33: 597-604, 2002.

48. Chen Y, Zhu J and Zhang W: Antitumor effect of traditional Chinese herbal medicines against lung cancer. Anticancer Drugs 25: 983-991, 2014.

49. Gillissen B, Wendt J, Richter A, Richter A, Müer A, Overkamp T, Gebhardt N, Preissner R, Belka C, Dörken B, et al: Endogenous Bak inhibitors Mcl-1 and Bcl-xL: Differential impact on TRAIL resistance in Bax-deficient carcinoma. J Cell Biol 188: 851-862, 2010.

50. Johnson TR, Stone K, Nikrad M, Yeh T, Zong WX, Thompson CB, Nesterov A and Kraft AS: The proteasome inhibitor PS-341 overcomes TRAIL resistance in Bax and caspase 9-negative or Bcl-xL overexpressing cells. Oncogene 22: 4953-4963, 2003. 
51. Lemke J, von Karstedt S, Abd El Hay M, Conti A, Arce F, Montinaro A, Papenfuss K, El-Bahrawy MA and Walczak H: Selective CDK9 inhibition overcomes TRAIL resistance by concomitant suppression of cFlip and Mcl-1. Cell Death Differ 21: 491-502, 2014

52. Lirdprapamongkol K, Sakurai H, Abdelhamed S, Yokoyama S, Athikomkulchai S, Viriyaroj A, Awale S, Ruchirawat S, Svasti J and Saiki I: Chrysin overcomes TRAIL resistance of cancer cells through Mcl-1 downregulation by inhibiting STAT3 phosphorylation. Int J Oncol 43: 329-337, 2013.

53. Siegelin MD, Gaiser T, Habel A and Siegelin Y: Daidzein overcomes TRAIL-resistance in malignant glioma cells by modulating the expression of the intrinsic apoptotic inhibitor, bcl-2. Neurosci Lett 454: 223-228, 2009.

54. Yoon MJ, Kang YJ, Kim IY, Kim EH, Lee JA, Lim JH, Kwon TK and Choi KS: Monensin, a polyether ionophore antibiotic, overcomes TRAIL resistance in glioma cells via endoplasmic reticulum stress, DR5 upregulation and c-FLIP downregulation. Carcinogenesis 34: 1918-1928, 2013.

55. Zang F, Wei X, Leng X, Yu M and Sun B: C-FLIP(L) contributes to TRAIL resistance in HER2-positive breast cancer. Biochem Biophys Res Commun 450: 267-273, 2014.
56. Darnell JE Jr: STATs and gene regulation. Science 277: 1630-1635, 1997.

57. Levy DE and Darnell JE Jr: Stats: Transcriptional control and biological impact. Nat Rev Mol Cell Biol 3: 651-662, 2002.

58. Bowman T, Garcia R, Turkson J and Jove R: STATs in oncogenesis. Oncogene 19: 2474-2488, 2000.

59. Bromberg J and Darnell JE Jr: The role of STATs in transcriptional control and their impact on cellular function. Oncogene 19: 2468-2473, 2000.

60. Buettner R, Mora LB and Jove R: Activated STAT signaling in human tumors provides novel molecular targets for therapeutic intervention. Clin Cancer Res 8: 945-954, 2002.

61. Song JI and Grandis JR: STAT signaling in head and neck cancer. Oncogene 19: 2489-2495, 2000.

62. Yu H and Jove R: The STATs of cancer - new molecular targets come of age. Nat Rev Cancer 4: 97-105, 2004.

63. Siveen KS, Sikka S, Surana R, Dai X, Zhang J, Kumar AP, Tan BK, Sethi G and Bishayee A: Targeting the STAT3 signaling pathway in cancer: Role of synthetic and natural inhibitors. Biochim Biophys Acta 1845: 136-154, 2014. 